\title{
Proposal for a solar fruit dryer design with organoleptic properties recovery system
}

\author{
Asmat-Campos, D. ${ }^{1,2}$, Angel Carreño-Ortega ${ }^{2}$ \\ ${ }^{1}$ Private University of North (UPN), Trujillo, Perú \\ ${ }^{2}$ Department of Engineering, Higher School of Engineering, Agri-Food Campus of International Excellence (CeiA3), \\ University of Almería, Ctra. Sacramento s/n, 04120 Almería, Spain.
}

\begin{abstract}
With the passing of time, the demand for dehydrated organic products has increased, ranging from vegetables to fruits, however in the latter efforts to achieve a quality product have led to the use of industrialized systems which depend on the use of fossil fuels or systems that use electric current which greatly increase production costs. At present, various solar dryer models have emerged, having achieved an optimal performance in what corresponds to the processing of vegetables, however in the processes of dehydration of fruits the results have not been satisfactory, due to the loss of its color, taste, aroma and texture (organoleptic properties). Faced with this problem, an innovative design of solar fruit dehydrator is proposed, which has two objectives, the first related to recovering the organoleptic properties through a system of condenzation, cyclic dehydration and the synchronization of the calorific balance, and the second one focused on the improvement of thermal efficiency, through the use of some heat piles and a trombe wall. The heat transfer fluid was improved, adding silver nanoparticles (silver nanospheres), which were synthesized using the green chemistry method; the efficiency of the heat transfer fluid was increased by $30 \%$.
\end{abstract}

\section{Introduction}

The drying process occurs naturally, an example of this is when the seeds are dried to reduce their mass and can be lighter for dispersion in the air. The key strategic objective in crop preservation is to reduce the number of undernourished people in the wider world [1]. Since past times, open-air drying has been practiced, where it consists only of exposing the harvested product to solar radiation, either by placing it on the ground or a mat, however, the product is prone to great losses, due to an uneven removal of moisture, and exposure to insects and rodents [2]. Dehydration is the most common form of food preservation, because it extends the life of food [3]. Water is the main component present in fruits, which is why it is essential to be able to control it in order to avoid organic and nutrient deterioration [4]. With a system of dehydration it is possible to preserve any type of organic material, this by means of the reduction of the levels of humidity, so that the microorganisms can not develop [5]. At present there are many artisanal ways of drying fruits using the sun, because it is considered a fairly cheap method [6].

In developing countries more than $80 \%$ of organic foods are produced by small farmers [7], however, they do not use solar drying or dehydration because they can not achieve a quality product, with several disadvantages in terms of of product competitiveness. There are several studies where they have shown that solar dryers can be effective [6].

The success in solar drying processes comes from transforming products such as vegetables which do not need much of a meticulous process in order to not lose their organoleptic properties. In the fruit export process, it happens that, depending on the refrigeration system, the cold line is sometimes not adequate, leading to losses that reportedly reach $21 \%$, while other sources estimate a value between 40 and $50 \%$ [8].

The process of dehydration is of the dual type, since it consists of the transfer of heat to the product located in a dehydration chamber, and the massive removal of moisture from the product from the interior to the exterior (surface) of the environment [9]. As in drying, dehydration leads to greater preservation of the product, becoming a method that manages to eliminate a large percentage of moisture content, however, there are few systems that achieve a product that meets quality standards, even more in the case of fruits, because the processes of heat transfer are not taken into account gradually, which leads to the loss of organoleptic properties. For years, systems and processes have been innovating under the implementation of technologies that depend on fossil fuels and / or the use of conventional electric energy by boilers, which have increased production costs by a large percentage, making it difficult for the consumer to be able to acquire it. 
There is a diversity of solar drying systems, which have been innovated through better heat transfer by convection and radiation, however, they have not been successful in relation to products with high fructose content, however, several researchers have developed the so-called solar dehydrators, which present a greater efficiency at thermal level, which became a much more viable option in comparison with high technology systems [10].

In this article, an innovative design of solar dehydrator is proposed, the same one that has two objectives, the first related to the preservation of the organoleptic properties in the process of dehydration of the fruit through the use of a system of recovery of aroma in the process of synchronized condensate and heat reflux, and secondly, the use of auxiliary backup systems to supply synchronized heat in periods of low intensity of solar radiation, in addition to increasing the heat transfer efficiency of the heat transfer fluid, by using of nanofluid based on silver nanoparticles, the same ones that were synthesized as part of this work, under a method denoted as green chemistry.

\section{Methodology}

In the agro-export market, the preservation of the product plays a fundamental role, having to use industrial refrigeration systems in order to achieve a product in good condition at the destination. Dehydration is an accepted alternative for large exporters, because a large range of fruits are accepted by the consumer in this presentation and in addition to modern freeze drying systems have achieved a product of excellent quality, however production costs they increase abysmally, that is why the proposal to use solar energy through an innovative system, would be efficient at a cost - benefit level, for this it is essential to establish the various variables that will intervene in the process, including relative humidity, temperature, volume of air and heat given.

Theoretically, it has been established that the synchronized increase of the heat transferred to the product leads to a gradual process of dehydration of the product, thus generating the non-rupture at the cellular level of the fruit, in turn the condensation system of the moisture of the product and the reflux of the product. heat transferred to complete the process, without suffering the sudden change of heat. In addition, the increase of heat through alternative heat sources to the system would generate an improvement in the efficiency of the equipment, however it is essential that the dehydration process be continuous (even in hours where there is no solar radiation), which is why it has been taken into account the adherence of a system called
"Thermobank", which consists of some thermal batteries which store heat and transmit them by convection and radiation to achieve a thermal equilibrium in hours where the temperature tends to fall.

\subsection{Dehydration equipment:}

The innovated model, presents simultaneously systems which generate a heat transfer, which are controlled by temperature and humidity sensors, and a moisture condensation system, which guarantee a good dehydration process and therefore a good product quality. In figure 1, the design of the equipment is appreciated. In the figure it can be seen that the system is composed of heat sources such as a parabolic cylindrical collector (by heating a high density fluid) reserving that heat in a thermobank, and a "trombe" wall, installed in one of the the rear faces of the dehydration chamber. Regarding the system of recovery of aromas and organoleptic properties, it consists of a system of condensation and drainage of moisture, recovering directly by means of a closed circuit assisted by a centrifugal fan, the aroma of the dehydration chamber. In the latter, the circuit has a series of passage valves, which are synchronized according to the process. The design also covers the accumulation of thermal energy for hours where solar radiation is insufficient.

\subsection{Process of operation:}

The designed equipment is specific for the dehydration of fruits by means of a system of calorific contribution assisted by two sources (trombe wall and parabolic cylindrical collector), It should be noted that this proposal is part of a patent by the authors of this research paper (Patent $\mathrm{N}^{\circ} \mathrm{P} 201731235$ ), in turn a thermobank is adapted to achieve the calorific balance by reducing the high temperatures obtained in parabolic cylindrical collector (when the intensity of solar radiation is high) and / or as a supplement to the reserve of heat energy in hours where solar radiation is weak (using heat piles, stones).

Inside the dehydration chamber there is a duct with an outlet in the upper part of the dehydration chamber and an entrance in the lower part of the chamber. It consists of three valves which act synchronously to let the moisture of the product pass, condensing it, draining it and finally return it to the system, all of it forced by a centrifugal fan, thus avoiding the loss of the aroma of the product (fruit) and other organoleptic properties; the system depends on two fundamental variables: temperature and humidity, for this case they will be 


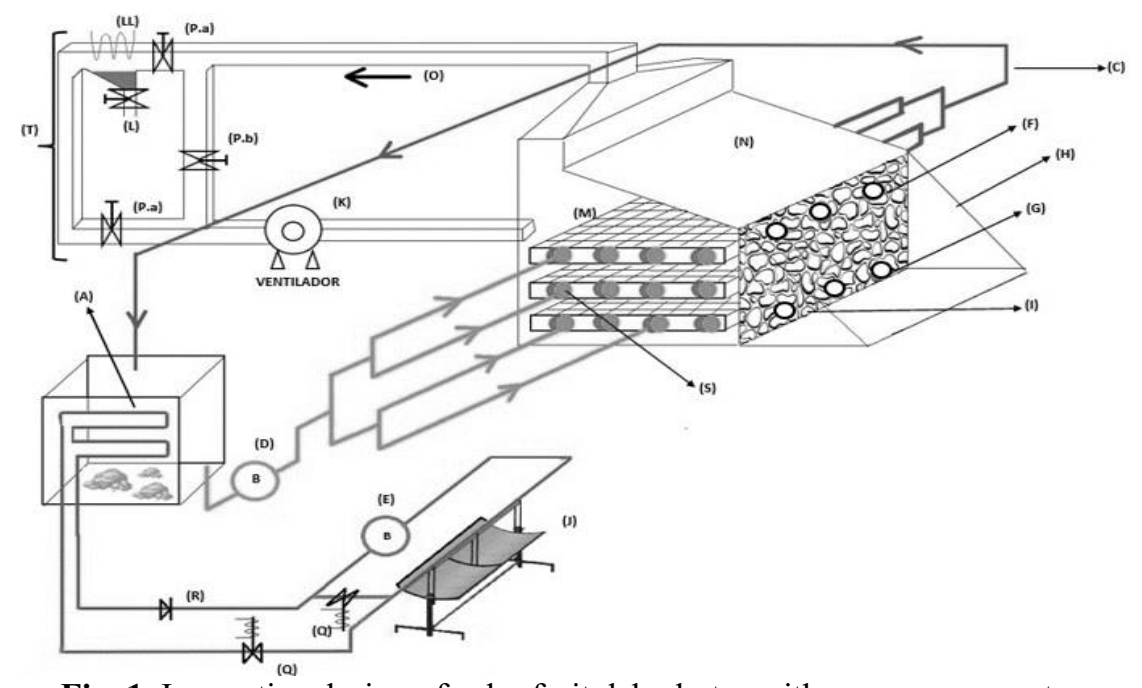

Fig. 1. Innovative design of solar fruit dehydrator with aroma recuperator.

monitored by humidity and temperature sensors with data logger, provided with electric current by photovoltaic panels.

The calorific contribution that has the dehydration chamber is given by two coupled systems, the first: The trombe wall (wall made of stones joined with concrete, with holes in the top and bottom, with the front side painted matt black, At the same time, on the outside, a fully waterproofed glass house to achieve a greenhouse effect and save heat in the thermal stone piles) (Figure 2), this system provides hot air by natural convection to the dehydration chamber (with greater intensity in hours where there is direct solar radiation and with less intensity where there is no solar radiation -night). Regarding the second source of heat input: The parabolic cylindrical collector is provided with the passage of an oil (heat transfer fluid) through the focal axis (which will act with a whole sequence of solenoid and check valves); however, the temperature is balanced by adapting a thermobank, which contains some stones (thermal batteries), passing to heat a second heat transfer fluid (water), being transported by copper pipes (properly thermally insulated) for conduction to the dehydration chamber, which provides the product with heat by thermal radiation; the fluid once transferred its calorific contribution to the product, will return to the thermobank (by natural convection) for its subsequent heating, working by means of a closed circuit.

The dehydration chamber has metal mesh trays placed one on top of the other, and in the lower part passes copper pipe where the second heat transfer fluid flows, thus providing an ideal temperature; At the same time, the calorific contribution of the trombe wall described above is added, thus improving the production speed of the dehydrated fruit.

The moisture emanating from the fruits as an effect of the dehydration process is conducted by the duct described above gradually, this process will be by forced draft, by coupling an air pump.

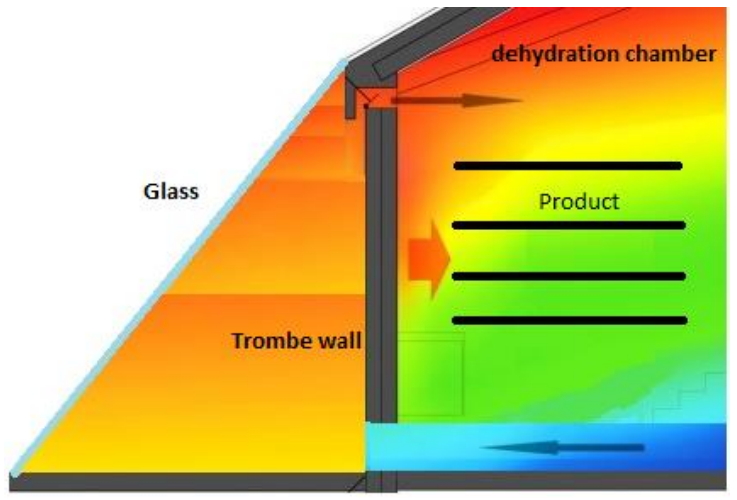

Fig. 2. Heat transfer through the trombe wall

This system solves the typical technical problem of solar dehydrators, such as inefficiency with respect to production time, counting with two sources of heat input, being able to work in high, medium and low intensity solar radiation conditions; However, it also highlights what relates to the recovery of aroma, because it is an important feature of arduous demand in export processes of dehydrated products or flour.

\subsection{Explanation of the innovative solar dehydration equipment}

Solar dehydrator fruit equipment, with aroma recovery system. (Figure 3 ).

The design consists of two structural complexes, which in turn raise two objectives, the first to recover the organoleptic properties of the fruit (specifically the aroma), and the second is to provide an efficient calorific contribution from solar energy, this last able to provide heat energy even at daytime where it is cloudy and / or at night. 


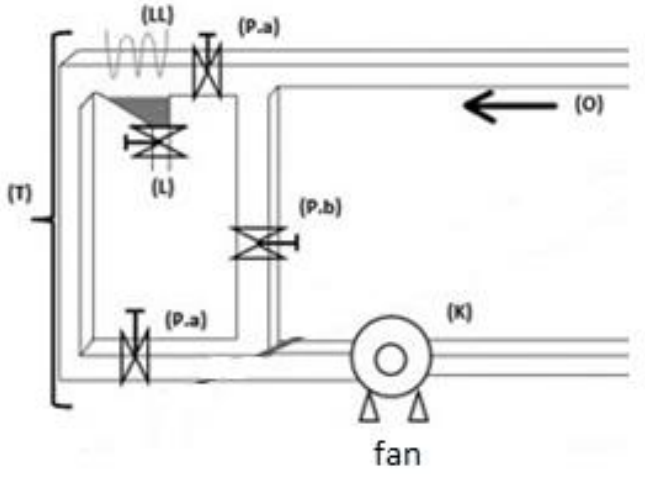

Fig. 3. Design of the organoleptic properties recovery system.

For the system of aroma recuperator (T), it will be able to capture the humidity coming from the fruit, coming from a first outlet pipe $(\mathrm{O})$, going through some valves $(\mathrm{Pa}) \mathrm{Y}(\mathrm{Pb})$, which will synchronize the passage of the humidity, until arriving at the condensation process by assisted conventional refrigeration (LL), and drainage (L); the process will be forced by centrifugal fan $(\mathrm{K})$. Secondly, with regard to the efficient calorific contribution, a design of thermal bank of heat or "thermobank" (A) has been incorporated in a traditional way, which allows under a conduction heat transfer process (heat emitted by pipeline). (E) coming from the parabolic cylindrical collector $(\mathrm{J})$ to the water of the thermobank (A) for its later conduction through the pipe (D) to the dehydration chamber allowing to "calibrate" the ideal temperature and in turn save the heat energy in some "Heat batteries" (stones) located in the thermobank, the latter with the objective that the dehydrator work in hours where there is no intensity of solar radiation (night) and / or this cloudy (low intensity of solar radiation), and emit its heat energy due to the effect of thermal equilibrium (zero law of thermodynamics) with the same destination (dehydration chamber), thus achieving the presence of controlled heat in the product that is e $\mathrm{n}$ a constant dehydration process.

\subsection{Description of the drawings:}

Figure 1. Solar dehydrating fruit equipment with aroma recovery system.

(A) Thermobank, (T) Scent recovery system, (C) Duct outlet (cold water) from the dehydrator, (D) Duct entrance with hot water to the dehydrator, (E) Piping with heat transfer fluid (oil) from the parabolic solar collector, (F) Hot air inlet holes, (G) Cold air outlet holes, (H) Glass hut with tilt angle, (I) Wall of stones joined with concrete, $(\mathrm{J})$ Parabolic cylindrical collector

(K) Centrifugal fan, (L) Humidity drainage, (LL) Humidity condenser per conventional refrigeration cycle, (M) Metal mesh trays, (N) Solar dehydration chamber, (O) Main pipeline, (P.a), (P.b) Valves, (Q) Solenoid valves, (R) Valve check, (S) Copper piping (hot water passage).

The modeling of the innovative design was made through the analysis of a native fruit of Peru called lúcuma (Pouteria lúcuma), with the aim of giving it an added value, due to the great international demand. For this case, the calculations related to the useful solar radiation were made by means of the equation (1) foreseen for the city of Trujillo, place that owns part of the greater production of this fruit, the city in study has a great agroexporter potential, being its favorable weather conditions (minimum) to be able to bet on applying the system envisaged. Trujillo (department of La Libertad) is located $34 \mathrm{msnm}$, in the north coast of Peru, taking into account that the average solar radiation intensity is 550 $\mathrm{W} / \mathrm{m}^{2}$, and its temperature varies between $18{ }^{\circ} \mathrm{C}$ and $30{ }^{\circ} \mathrm{C}$ in winter and summer respectively. It should be noted that in Peru there are areas in the sierra area, whose intensity of solar radiation reaches up to $1700 \mathrm{~W} /$ $\mathrm{m}^{2}$, additional motivation that was taken with the aim of achieving the same thermal conditions to achieve dehydration of products with total normality.

$$
S=\left(I_{t}\right)(k)(\tau)\left(\alpha_{b}\right)
$$

The results showed that for the study area the useful solar radiation with which it would be counted (on average per year) is $390.45 \mathrm{~W} / \mathrm{m}^{2}$, in addition to this there is the calculation related to the useful power, both for the wall system trombe the heating of an air volume of $13.53 \mathrm{~m}^{3}$, and a concrete trombe wall with masses of stones, achieving a value of $202.22 \mathrm{~W} / \mathrm{h}$ and $420 \mathrm{~W} / \mathrm{h}$. respectively; The following equations are those used for the respective calculations of the useful power of the trombe wall and the parabolic cylindrical collector (CCP) respectively.

$$
\begin{gathered}
Q_{u}=\left(A_{c}\right)\left(F_{R}\right)\left[(S)-U_{L}\left(T^{\circ}{ }_{f i}-T^{\circ}{ }_{a}\right)\right] \\
q=\frac{T-T_{\infty}}{\frac{\ln r_{3} / r_{2}}{(2)(\pi)(l)\left(K_{v}\right)}+\frac{1}{(h)(2)(\pi)(l)\left(r_{3}\right)(180 / 360)}}
\end{gathered}
$$

As part of the mathematical analytical protocol of the calculations, it was also taken into account, for the case of the CCP, the calculation of the geometric concentration ratio, the solar energy incident on the collector and the amount of useful thermal energy supplied by the equipment in mention.

\subsection{Improvement of the efficiency of the heat transfer fluid, used nanoparticles}

The dehydration system will have as energy sources both the trombe wall and the parabolic cylindrical collector, for the latter it is necessary to have a heat transfer flow, that is why the oil is used, however it is planned to add silver nanoparticles to the oil (silver nanospheres) the same ones that were chemically synthesized using silver nitrate $\left(\mathrm{AgNO}_{3}\right)$ as a precursor agent, and reduced using natural extract of the blueberry fruit, which makes it a green and non-polluting synthesis process. The results have been quite favorable when using this organic agent as a reducer, achieving nanospheres with a diameter that are within the range of 15 to $60 \mathrm{~nm}$. Characterizations 
were made to the nanoparticles by X-ray diffraction, showing the peaks belonging to the crystalline structure of the silver, dynamic light scattering (DLS), which showed the diameters of the nanospheres, the latter corroborated by TEM images, to its Once spectrophotometry was performed, resulting in an optical absorbance peak of $420 \mathrm{~nm}$ (Figure 4), typical of spherical silver nanostructures.

The adhesion of this type of material to the heat transfer fluid improves a thermal efficiency of $30 \%$.

The system of dehydration in general, consists of two thermal sources, in addition the adhesion of a thermobank (thermal bank), adds an efficiency in the productive process, increased the hours in which the product will be exposed to heat and carrying out the process dehydrated, the synchronization of the heat transfer is the key to carry out a moderate process of evaporation of the humidity of the fruit, adding a process of condensation of moisture and drained of it under processes already planned, stage which will have as objective not to lose the organoleptic properties of the fruit; the solar panels will be a voltage source for thermometric and humidity sensors.

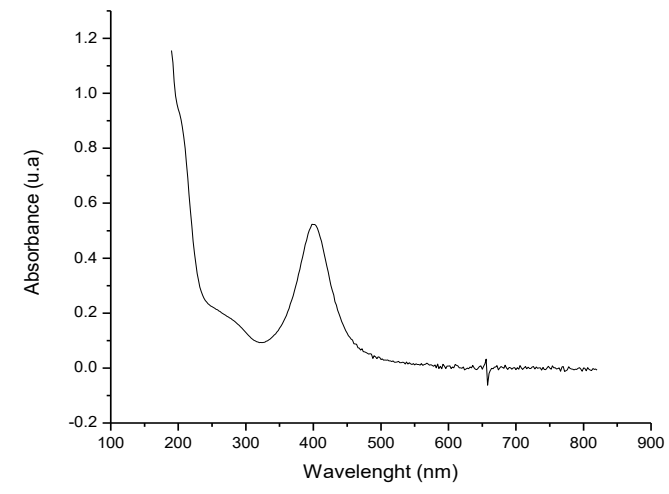

Fig. 4. Optical absorbance graph of the organic silver nanoparticles.

\section{Conclusions}

The innovative model of solar dehydrator, was able to form a hybrid system (cylindrical parabolic collector, wall trombe and thermobank), which add an energy of $622.22 \mathrm{w} / \mathrm{h}$., and with daily dehydration capacity of 4.38 $\mathrm{kg}$. of raw mass and $1.74 \mathrm{~kg}$. of flour per day, being able to increase its capacity according to the area of the dehydration chamber; it would also reduce production costs by $23 \%$ compared to a conventional electric dehydrator; the benefit would also be reflected in the environmental field, reducing a total of $444.27 \mathrm{~kg}$. of annual co2 with only one team, which is why the project could benefit from carbon credits. The use of silver nanofluids, increased the thermal conductivity capacity by $30 \%$.

\section{References}

1. Anon., 2004. The State of Food Insecurity in the World, Food and Agriculture Organization of the United Nations Viale delleTterme di Caracalla, 00100 Rome, Italy.

2. Tomar, V., et al. Solar dryers for tropical food preservation: Thermophysics of crops, systems and components. Sol. Energy (2017).

3. Sarasavadia, P.N., Sawhney, R.L., Pangavhane, D.R., Singh, S.P., 1999. Drying behaviour of brined onion slices. Journal of Food Engineering 40, 219 226.

4. Saguy, I., Karel, M., 1980. Modelling of quality deterioration during food processing and storage. Food Technology 78, 84.

5. Mahmutoglu, Teslime, Ferhunde, Emir, Birol Saygi, Y., 1996. Sun/solar drying of differently treated grapes and storage stability of dried grapes. Journal of Food Engineering 29 (3-4), 289-300.

6. Pangavhane, D.R., Sawhney, R.L., 2002. Review of research and development work on solar dryers for grape drying. Energy Conversion and Management 43, 45-61.

7. Murthy, M.V.R., 2009. A review of new technologies, models and experimental investigations of solar driers. Renewable and Sustainable Energy Reviews 13 (4), 835-844.

8. Sodha, M.S., Bansal, N.K., Kumar, Ashvini, Bansal, P.K., Malik, M.A.S., 1986. Solar Crop Drying. CRC Press, New York.

9. Ekechukwu, O.V., Norton, B., 1999. Review of solar-energy drying systems II: an overview of solar drying technology. Energy Conversion and Management 40, 615-655.

10. A. Sharma, C.R. Chen y Nguyen Vu Lan. "Solar energy drying systems: A review". Renewable and sustainable energy reviews, vol 13 (2009), pp.1185 -1210 .

11. O. V. Ekechukwu. "Review of solar-energy drying systems III: low temperature air-heating solar collectors for crop drying applications". Energy Conversion and Management, vol 40, No 6. (1999), pp.657-667.

12. O. V. Ekechukwu. "Review of solar-energy drying systems I: an overview of drying principles and theory”. Energy Conversion \& Management, vol 40, No 6. (1999), pp. 593613.

13. Ali Motaveli, Saeid Minaei y Mohammad Hadi Khoshtagaza. "Evaluation of energy consumption in different drying methods". Energy conversion and management, vol 52, (2011), pp. 1192 - 1199.

14. F. Rodríguez. Ingeniería de la industria alimentaria (III). Conservación de alimentos por reducción de su actividad de agua. Secado. Capítulo 5, 2002.

15. I. Doymaz. "Convective drying kinetics of strawberry". Journal Chemical engineering and processing, vol 47 (2008), pp. 914-91 